\title{
Pricing Equity Default Swaps under an approximation to the CGMY Lévy Model
}

\author{
S. Asmussen \\ Dept. of Mathematical Sciences \\ Aarhus University \\ Ny Munkegade \\ DK-8000 Aarhus C, Denmark \\ D. Madan \\ M. Pistorius \\ Robert H. Smith School of Business \\ Dept. of Mathematics \\ Van Munching Hall \\ University of Maryland \\ College Park, MD 20742 \\ King's College London \\ Strand \\ London WC2R 2LS \\ U.K.
}

October 29, 2018

\begin{abstract}
The Wiener-Hopf factorization is obtained in closed form for a phase type approximation to the CGMY Lévy process. This allows, for the approximation, exact computation of first passage times to barrier levels via Laplace transform inversion. Calibration of the CGMY model to market option prices defines the risk neutral process for which we infer the first passage times of stock prices to $30 \%$ of the price level at contract initiation. These distributions are then used in pricing $50 \%$ recovery rate equity default swap (EDS) contracts and the resulting prices are compared with the prices of credit default swaps (CDS). An illustrative analysis is presented for these contracts on Ford and GM.
\end{abstract}

Acknowledgement 1 This work was partly completed while all three authors were visiting the Isaac Newton Institute as participants in the "Developments in Quantitative Finance" program. We thank Bruno Dupire and other seminar participants at Bloomberg. We also thank conference participants of the Credit Conference at Edinburgh and the MSRI conference in London.

Acknowledgement 2 In addition Martijn Pistorius acknowledges support from the Nuffield Foundation Grant NAL/00761/G. 


\section{Introduction}

The equity default swap contract has recently been introduced in the financial markets. The contract pays a recovery proportion of a notional amount at the time of the occurence of a specific equity event, up to a maturity, that is typically 5 years. The equity event is defined as the first time the stock price drops below $30 \%$ of the price prevailing at contract initiation. The distribution of this first passage time is then of critical importance in the valuation of the contract. These distributions are known for the geometric Brownian motion model and results have also recently been obtained (Davydov and Linetsky (2001), Campi and Sbuelz (2005)) for the constant elasticity of variance (CEV) model. The prices of European options under these models, however, are not consistent with observed market option prices and such observations call into question the resulting swap prices.

Observed market option prices are more closely matched by a variety of pure jump Lévy process models for the evolution of the logarithm of the stock price. Here we shall focus attention on the four parameter CGMY Lévy model introduced by Carr, Geman, Madan and Yor (2002). First passage times for such Lévy processes require knowledge of the distribution of the supremum and infimum of the process over a fixed time interval. The product of the Laplace transforms of these distributions is known by the famed Wiener-Hopf factorization. Identification of the law of the infimum and supremum then flounders on the inability to analytically perform the factorization. Again, it is known that if the Lévy process has only one-sided jumps, say downwards, then the law of the supremum is known and one may then use the Wiener-Hopf identity to deduce the law of the infimum. Such strategies have been effectively pursued in Rogers (2000), Novikov, Melchers, Shinjikashvili and Kordzakhia (2003), Khanna and Madan (2004) and Chiu and Yin (2005). Processes with one sided jumps are dominated by those with two sided jumps in their ability to explain option prices.

For Lévy processes with two sided jumps the double Laplace transform in time and the level of the infimum and supremum have been derived by Nguyen and Yor (2002) for some Lévy processes using bivariate integral representations. However, the numerical inversion of these transforms involves four integrals and is computationally quite involved. Here we consider an alternative strategy for obtaining the first passage distribution of two sided jump Lévy processes. To this end, we use phase-type distributions, defined as the time until absorption of a finite state continuous-time Markov process (See Asmussen (1992)). It is a well known fact that this class comes close to being complete for obtaining first passage probabilities of the type we consider.

We employ results from Asmussen, Avram and Pistorius (2004) to obtain closed forms for the Laplace transform of first passage distributions for Lévy processes when the Lévy measure has a phase type distribution. In addition to providing tractable computational schemes, phase-type distributions have the advantage of forming a dense class so that by increasing the size of the state space, one can in principle get arbitrarily close to a target density and thereby 
get arbitrarily good approximations of the first passage probabilities.

A particularly important subclass of phase-type distributions is formed by the hyperexponential distributions, i.e. finite mixtures of exponentials. The underlying Markov process then chooses some initial state, stays there an exponential time with rate depending on the state and goes directly to the absorbing state. This subclass is appealing for the $C G M Y$ Lévy case as the target density is completely monotonic, i.e. a possibly continuous mixture of exponentials, so that by a discretization procedure it is a limit of (possibly scaled) hyperexponential densities. In practice, the fitting can be done either by maximum likelihood (Asmussen, Nerman and Olsson (1996)) as then one wants to capture the shape of the target density, or by minimizing a distance measure paying specific attention to specific features of interest. In particular, in first passage problems a good fit in the tail is crucial, and hyperexponential approximations to Pareto tails have been obtained in this way (Feldman and Whitt (1998)). This is the path we follow in this paper. We approximate the Lévy density of the $C G M Y$ process by a hyperexponential distribution and we then use the first passage time of the approximating process to determine the Laplace transform of the first passage time distribution for the approximating Lévy process. One dimensional Laplace transform inversions using the methods of Abate and Whitt (1995) then give us both the survival probability and the first passage density for this approximation. One may wish to evaluate the quality of this approximation to an exact result for the CGMY process. The latter is however not computationally available at this time. One may obtain alternative approximations by either solving partial integro differential equations or by Monte Carlo simulation. The simulation would require the development of appropriate importance sampling methodologies. We therefore leave these questions for future research. The same considerations apply with respect to constructing a priori bounds on the error with respect to the exact result.

From the first passage density and survival probability one may determine the quote on an equity default swap contract. We estimate the CGMY parameters from the prices of options on Ford and GM over a three year period and extract daily estimates of quotes on equity default swap contracts using the above hyperexponential approximation. We compare these prices with market quotes on the $C D S$ rates and we observe that these two separate sets of prices for a credit event, indeed, correlate well.

The outline of the rest of the paper is as follows. Section 2 presents the details for the construction of the Laplace transform of the first passage time for an approximation to the CGMY Lévy process. Section 3 describes how this transform is used in quoting on equity default swap contracts. Section 4 presents the results of daily calibration of the $C G M Y$ model to market option prices. Section 5 compares the resulting equity default swap prices with their credit default swap counterparts. Section 6 concludes. 


\section{The CGMY First Passage Time}

We wish to determine the first passage time of the $C G M Y$ Lévy process to various levels using a phase type distribution to approximate the process. For the Laplace transform of the first passage time of the approximating process, i.e. a process with a phase type Lévy measure, we follow Asmussen, Avram and Pistorius (2004). Given that the Lévy measure is completely monotone we may employ a very special phase type distribution that approximates the Lévy measure as a mixture of exponentials. The phase type distribution we employ therefore has a simple form. Starting from an initial state we have an entrance into a number of states where we stay until absorption. There are no transitions between states. Suppose the Lévy measure has the form

$$
\begin{aligned}
k(x)= & \sum_{i=1}^{n} a_{i} e^{-\alpha_{i} x} \mathbf{1}_{x>0} \\
& +\sum_{j=1}^{m} b_{j} e^{-\beta_{j}|x|} \mathbf{1}_{x<0} .
\end{aligned}
$$

Our phase type distribution for the positive jumps has $n+1$ states with the generator matrix

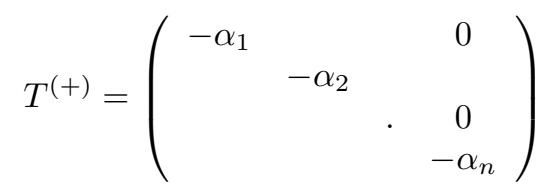

with unnormalized initial state probabilities proportional to $\frac{a_{i}}{\alpha_{i}}$ for the states $i=1, \cdots, n$ and the vector of absorption rates into the final state is

$$
t=\left(\alpha_{1}, \alpha_{2}, \cdots, \alpha_{n}\right) .
$$

The characteristic exponent for the approximation now takes the special form

$$
\begin{aligned}
\kappa(s) & =\log E\left[\exp \left(s X_{1}\right)\right] \\
& =\mu s+\lambda_{+} \sum_{i=1}^{n} \pi_{i}^{+}\left(\frac{\alpha_{i}}{\alpha_{i}-s}-1\right)+\lambda_{-} \sum_{j=1}^{m} \pi_{j}^{-}\left(\frac{\beta_{j}}{\beta_{j}+s}-1\right)+\frac{\sigma^{2}}{2} s^{2} \\
\lambda^{+} & =\sum_{i=1}^{n} \frac{a_{i}}{\alpha_{i}} ; \lambda_{-}=\sum_{j=1}^{m} \frac{b_{j}}{\beta_{j}} \\
\pi_{i}^{+} & =\frac{a_{i}}{\lambda_{+} \alpha_{i}} ; \pi_{j}^{-}=\frac{b_{j}}{\lambda_{-} \beta_{j}} .
\end{aligned}
$$

Where the value of $\sigma^{2}$ will depend on the diffusion approximation we incorporate for the small jumps. This is described in greater detail later. The drift $\mu$ is chosen to make the overall drift on the stock price be the interest rate less the dividend yield. 
Let $T_{x}$ be the first passage time over a level $x>0$, the Laplace transform of $T_{x}$ at the transform argument $a$

$$
E\left[e^{-a T_{x}}\right]
$$

is obtained in terms of the roots, with positive real part, of the equation

$$
\kappa(s)=a .
$$

We observe that one may write

$$
\kappa(s)=\frac{p(s)}{q(s)}
$$

as a ratio of two polynomials and so we seek the roots of the polynomial equation

$$
p(s)=a q(s) .
$$

The polynomial $q(s)$ is

$$
q(s)=\prod_{i=1}^{n}\left(\alpha_{i}-s\right) \prod_{j=1}^{m}\left(\beta_{j}+s\right)
$$

and

$p(s)=q(s)\left(\frac{\sigma^{2}}{2} s^{2}+\mu s-\left(\lambda_{+}+\lambda_{-}\right)-\lambda_{+} \sum_{i=1}^{n} \pi_{i}^{+} \alpha_{i} \frac{1}{s-\alpha_{i}}+\lambda_{-} \sum_{j=1}^{m} \pi_{j}^{-} \beta_{j} \frac{1}{\beta_{j}+s}\right)$.

We observe that $\kappa(0)=0$ and the poles of $\kappa(s)$ are exactly equal to the eigenvalues of $-T^{(+)}$and those of $T^{(-)}$(and also the roots of $q(s)=0$ ).We note that $p-a q$ is of degree $n+m+2$ if $\sigma>0$ and of degree $n+m+1$ if $\sigma=0, \mu>0$. In the case $\mu>0, \sigma=0, \kappa(s)=a$ has $n+1$ distinct positive roots and $m$ distinct negative roots. If $\sigma>0 \kappa(s)=a$ has $n+1$ distinct positive roots and $m+1$ distinct negative roots. Finally for $\mu=0, \sigma=0$ there are $n$ positive and $m$ negative distinct roots.

Let $k_{+}$be the number of positive roots. Suppose the positive roots are $\rho_{i}$, $i=1, \cdots, k_{+}$then letting

$$
M_{t}=\sup _{s \leq t}\left(X_{s} \vee 0\right)
$$

and writing

$$
P\left(M_{e(a)} \in d x\right)=\int_{0^{+}}^{\infty} a e^{-a t} P\left(M_{t} \in d x\right)
$$

$\left(e(a)\right.$ is an independent exponential random variable with mean $\left.a^{-1}\right)$, we have that (for $s$ with $\mathcal{R}(s) \leq 0$ ) 


$$
\begin{aligned}
\phi_{a}^{+}(s) & =\int_{0}^{\infty} a e^{-a t} E\left[e^{s M_{t}}\right] d t \\
& =P\left(M_{e(a)}=0\right)+\int_{0^{+}}^{\infty} e^{s x} P\left(M_{e(a)} \in d x\right) \\
& =\phi_{a}^{+}(-\infty)+\int_{0^{+}}^{\infty} e^{s x} P\left(M_{e(a)} \in d x\right) .
\end{aligned}
$$

This function may be expressed in terms of the positive roots $\rho_{i}$ by

$$
\begin{aligned}
\phi_{a}^{+}(s) & =\frac{\operatorname{det}(-s I-T)}{\operatorname{det}(-T)} \cdot \frac{\prod_{i=1}^{k_{+}}\left(-\rho_{i}\right)}{\prod_{i=1}^{k_{+}}\left(s-\rho_{i}\right)} \\
& =\frac{\prod_{i=1}^{n}\left(1-s \alpha_{i}^{-1}\right)}{\prod_{i=1}^{k_{+}}\left(1-s \rho_{i}^{-1}\right)}
\end{aligned}
$$

where $\rho_{1}, \cdots \rho_{k_{+}}$are the positive roots of $\kappa(s)=a$ and where $T=\operatorname{diag}\left(-\alpha_{i}\right)$ is the restriction of $T^{+}$to the nonabsorbing states. If $\sigma=0$ the number of positive roots $k_{+}$is equal to the number of positive phases $n, k_{+}=n$ and we note that

$$
\phi_{a}^{+}(-\infty)=\prod_{i=1}^{k_{+}} \frac{\rho_{i}}{\alpha_{i}}
$$

and $0<\phi_{a}^{+}(-\infty)<1$.

If $\sigma>0$ then the number of positive roots is $k_{+}=n+1$ one more than the number of positive phases $n: \rho_{1}, \cdots \rho_{n+1}$ and we have the formula

$$
\phi_{a}^{+}(s)=\frac{\prod_{i=1}^{n}\left(1-s \alpha_{i}^{-1}\right)}{\prod_{i=1}^{n+1}\left(1-s \rho_{i}^{-1}\right)}
$$

furthermore, now $\phi_{a}^{+}(-\infty)=\lim _{s \rightarrow-\infty} \phi_{a}^{+}(-s)$ is zero.

Hence in the case that there is a Brownian component present $(\sigma>0)$

$$
\phi_{a}^{+}(-\infty)=0 .
$$

By performing a partial fraction decomposition we can perform the Laplace inversion in $s$ analytically: Note that we can write

$$
\phi_{a}^{+}(s)-\phi_{a}^{+}(-\infty)=\sum_{i=1}^{k_{+}} A_{i}^{+} \frac{-\rho_{i}}{s-\rho_{i}}
$$

where

$$
A_{i}^{+}=\frac{\prod_{j=1}^{n}\left(1-\rho_{i} \alpha_{j}^{-1}\right)}{\prod_{j=1, j \neq i}^{k_{+}}\left(1-\rho_{i} \rho_{j}^{-1}\right)} .
$$


The Laplace transform can be inverted explicitly to find for $x>0$

$$
P\left(M_{e(a)}<x\right)=P\left(M_{e(a)}=0\right)+\sum_{i=1}^{k_{+}} A_{i}^{+}\left(1-e^{-\rho_{i} x}\right) .
$$

It follows from the fact that

$$
P\left(M_{e(a)}<\infty\right)=1
$$

that

$$
1-\sum_{i=1}^{k_{+}} A_{i}^{+}=P\left(M_{e(a)}=0\right)=\phi_{a}^{+}(-\infty) .
$$

In particular we get that

$$
\begin{aligned}
P\left(M_{e(a)}>x\right) & =1-P\left(M_{e(a)}=0\right)-\sum_{i=1}^{k_{+}} A_{i}^{+}\left(1-e^{-\rho_{i} x}\right) \\
& =1-\sum_{i=1}^{k_{+}} A_{i}^{+}-P\left(M_{e(a)}=0\right)+\sum_{i=1}^{k_{+}} A_{i}^{+} e^{-\rho_{i} x} \\
& =\sum_{i=1}^{k_{+}} A_{i}^{+} e^{-\rho_{i} x} .
\end{aligned}
$$

Now since

$$
\begin{aligned}
P\left(M_{e(a)}>x\right) & =\int_{0}^{\infty} a e^{-a t} P\left(M_{t}>x\right) d t \\
& =a \int_{0}^{\infty} e^{-a t} P\left(T_{x}<t\right) d t
\end{aligned}
$$

we find the Laplace transform of $P\left(T_{x}<t\right)$

$$
\int_{0}^{\infty} e^{-a t} P\left(T_{x}<t\right) d t=\frac{1}{a} \sum_{i=1}^{k_{+}} A_{i}^{+} e^{-\rho_{i} x}
$$

where $\rho_{i}=\rho_{i}(a)$ and $A_{i}^{+}=A_{i}^{+}(a)$ depend on $a$.

We employ the methods of Abate and Whitt (1995) to obtain first passage probabilities and the first passage density. We next consider the details for the diffusion approximation and the phase type approximation of the $C G M Y$ process.

\subsection{CGMY details}

The CGMY process was introduced in (Carr, Geman, Madan and Yor (2002), see also Koponen (1995), Boyarchenko and Levendorskii $(1999,2000)$ ) and is a 
pure jump Lévy process with the Lévy density

$$
k(x)=C \frac{e^{-M x}}{x^{1+Y}} \mathbf{1}_{x>0}+C \frac{e^{-G|x|}}{|x|^{1+Y}} \mathbf{1}_{x<0} .
$$

For this completely monotone Lévy density of the $C G M Y$ process we recognize that

$$
\frac{1}{x^{1+Y}}=\int_{0}^{\infty} \frac{u^{Y} e^{-u x}}{\Gamma(1+Y)} d u
$$

and consider the approximation scheme

$$
\frac{1}{x^{1+Y}} \approx \sum_{i=1}^{N-1} \frac{u_{i}^{Y} e^{-u_{i} x}\left(u_{i+1}-u_{i}\right)}{\Gamma(1+Y)} .
$$

For a prespecified sequence of exponential decay coefficients $u_{i}$ that correspond to reasonable levels of mean jump sizes under the single exponential model. The specific values for $u_{i}$ were obtained using a least squares algorithm that minimizes the sum of squared errors between the left hand side of (27) and the right hand side of (27) evaluated at the $x$ points starting at $x=.25$ and increasing to $x=5$ in steps of .025 . As starting values we employed $.5,2,5,10,20,40$ and 100. Our application works with the value of $Y$ fixed at .5 and hence the approximation of equation (27) developed and subsequently used is independent of all parameter variations. The resulting values for $u_{i}$ were $.1940, .5982, .8434,1.1399,1.5308,2.1211$, and 3.4055 .

We then approximate the $C G M Y$ Lévy density on the two sides by

$$
\begin{aligned}
k_{+}(x) & =\sum_{i=1}^{N-1} c_{i} e^{-\left(M+u_{i}\right) x} \\
k_{-}(x) & =\sum_{i=1}^{N-1} d_{i} e^{-\left(G+u_{i}\right) x} . \\
c_{i} & =d_{i}=\frac{C u_{i}^{Y}\left(u_{i+1}-u_{i}\right)}{\Gamma(1+Y)}
\end{aligned}
$$

We then have in terms of our general discussion above

$$
\alpha_{i}=M+u_{i} ; \beta_{i}=G+u_{i}
$$

Alternative but somewhat related procedures for fitting hyperexponentials to general densities (rather than Lévy measures as here) have been considered in Feldman and Whitt (1998), Andersen and Nielsen (1998) and Asmussen, Jobmann and Schwefel (2002). 


\subsection{First Passage to a low level}

For the first passage to a low level we may work with the first passage of $-X(t)$ to a high level. In this case we reverse the roles of $\alpha, \beta$ and write

$$
\begin{aligned}
& \alpha_{i}=G+u_{i} \\
& \beta_{i}=M+u_{i} .
\end{aligned}
$$

\subsection{The small jump diffusion approximation}

Above we discussed a procedure to approximate a $C G M Y$ process $X$ by a Lévy process $X_{1}$ with density (1) and to improve the approximation we would like now to approximate the difference by a Brownian motion.

A way to refine the above approximation of $X$ by $X_{1}$ is to approximate the process of small jumps by a Brownian motion. This process of small jumps denoted $Z_{1}^{\varepsilon}$ is obtained using the Lévy density of $X$ restricted to $(-\varepsilon, \varepsilon)$. The process $Z_{1}^{\varepsilon}$ is approximated by a Brownian motion with variance

$$
\sigma^{2}=\sigma^{2}(\varepsilon)=\int_{-\varepsilon}^{\varepsilon} x^{2} \nu(d x)
$$

In Asmussen and Rosinski (2001) it is shown that, if $\nu$ has no atoms, $Z_{1}^{\varepsilon}$ weakly converges to a Brownian motion if and only if

$$
\lim _{\varepsilon \rightarrow 0} \frac{1}{\varepsilon} \int_{-\varepsilon}^{\varepsilon} x^{2} \nu(d x)=\infty
$$

For a Variance Gamma process this diffusion approximation fails as

$$
\lim _{\varepsilon \rightarrow 0} \frac{1}{\varepsilon} \int_{0}^{\varepsilon} x e^{-a x} d x<\infty
$$

For a $C G M Y$ process with $Y>0$ this diffusion approximation is valid, since

$$
\lim _{\varepsilon \rightarrow 0} \frac{1}{\varepsilon} \int_{0}^{\varepsilon} x^{1-Y} e^{-a x} d x=\infty
$$

(as $\nu(d x) / d x \geq$ const $/|x|^{1+Y}$ in a neighbourhood of the origin, see Example 2.3 in Asmussen and Rosinski (2001)).

In our $C G M Y$ process approximation we truncated the $C G M Y$ Lévy density at $u_{1}=.5$ on the one hand but, on the other hand, the approximating densities $k_{+}(x), k_{-}(x)$ are taken to start at $x=0$. Therefore we apply the diffusion approximation to

$$
\widetilde{k}(x)=\left(e^{-M x} / x^{1+Y}-k_{+}(x)\right) \mathbf{1}_{0<x<u_{1}}+\left(e^{-G|x|} /|x|^{1+Y}-k_{-}(x)\right) \mathbf{1}_{-u_{1}<x<0} .
$$




\section{$3 \quad$ Equity Default Swap Pricing}

We essentially follow the logic for the pricing of credit default swaps, replacing the required survival probabilities and default time densities with the first passage complementary distributions $\bar{F}_{n}(C, G, M, Y)$ and the first passage time density $f_{n}(C, G, M, Y)$ as computed by Laplace transform inversion using the approximation methods described above in section 2 for $n$ days or time $360 t=$ $n$.

Like the credit default swap, the equity default swap contract is viewed in two parts, one describing the receipt side of the cash flows and the other the payment side. There is a notional amount $M$ associated with the receipt side and the actual level of cash flows received is $M$ times one minus the recovery rate $R$ on the occurence of the equity event. Our calculations employ a recovery rate of $50 \%$. These funds are received on the time $\tau$ of first passage of the equity to a level below the specified barrier. We employ two barriers in our calculations, these are $50 \%$ and $30 \%$ of the price at initiation.

Against this stream of receipts the equity default swap holder makes periodic coupon payments, typically until the equity event or the maturity whichever is comes first. At the first passage event date we subtract from the receipts the accrued coupons from the last coupon date before $\tau$ to this date. We denote time measured in days by $n$ and in years by $t$. For each day $n$ we define the function $\zeta(n)$ that gives the number of days between the end of day $n$ and the last day on which a coupon was paid. We also denote by $N$ the maturity of the contract in days while $T$ is the maturity in years. By convention we take $(N, n)=360(T, t)$.

Let $\Delta(n)$ be the first passage event indicator function that takes the value 1 if the first passage of equity has occurred on or before day $n$ and is zero otherwise. Further, let $k_{T}$ denote the annual coupon rate or the equity default swap rate quoted on the contract for maturity $T$. The cash flow receipts on day $n, R_{n}$ are then written as

$$
R_{n}=(\Delta(n)-\Delta(n-1))\left(M(1-R)-\frac{M k_{T}(n-\zeta(n))}{360}\right) .
$$

Let $N P$ denote the number of coupon payment dates and let $n p_{j}$ be the day number of the the $j^{\text {th }}$ payment date. The $j^{\text {th }}$ payment $P_{j}$ occurring on day $n p_{j}$ is then given by

$$
P_{j}=\frac{M k_{T}\left(n p_{j}-n p_{j-1}\right)}{360}\left(1-\Delta\left(n p_{j}\right)\right),
$$

where the multiplication by the complementary first passage event indicator function recognizes that no coupon payments are made after the first passage event.

For the valuation of claims we employ a discount function $B_{n}$ that gives the present value of a dollar promised on day $n$. There are a variety of approaches to constructing such discount functions. 
The random present value of cash flows to the equity default swap contract, $V(T)$ is then given by

$$
V(T)=\sum_{n=1}^{N} R_{n} B_{n}-\sum_{j=1}^{N P} P_{j} B_{n p_{j}} .
$$

We are supposing here that conditional on the outcome of the first passage event the expected values of future dollars are the same. This is equivalent to supposing that the first passage events of single names in the economy do not contain any information about macro movements in interest rates or that interest rate evolutions are independent of the first passage process. We leave for future research the modeling of joint evolutions of interest rates and first passage times.

The equity default swap quotes in markets are set at levels consistent with a zero price at the initiation of the swap contract. Hence we have that under the risk neutral measure

$$
E^{Q}[V(T)]=0 .
$$

From our risk neutral distribution for the first passage time we employ

$$
E^{Q}[(1-\Delta(n))] \underset{D e f}{=} \bar{F}_{n}(C, G, M, Y) .
$$

The probability of first passage on a particular day $n$ may be approximated by the density of default times the length of the day in years.

$$
\begin{aligned}
& E^{Q}[(\Delta(n)-\Delta(n-1))] \underset{\text { Def }}{=} \pi_{n}(C, G, M, Y) \\
\approx & f_{n}(C, G, M, Y) \frac{1}{365} .
\end{aligned}
$$

We may then compute the value of the equity default swap quote as

$$
\begin{aligned}
E^{Q}[V(T)]= & \sum_{n=1}^{N}\left[M(1-R)-\frac{M k_{T}(n-\zeta(n))}{360}\right] B_{n} \pi_{n}(C, G, M, Y)- \\
& \sum_{j=1}^{N P} \frac{M k_{T}\left(n p_{j}-n p_{j-1}\right)}{360} B_{n p_{j}} \bar{F}_{n p_{j}}(C, G, M, Y) .
\end{aligned}
$$

Setting this value to zero and solving for the $k_{T}$ we obtain the $C G M Y$ equity default swap pricing model

$k_{T}(C, G, M, Y)=\frac{(1-R) \sum_{n=1}^{N} B_{n} \pi_{n}(C, G, M, Y)}{\sum_{j=1}^{N P} \frac{\left(n p_{j}-n p_{j-1}\right)}{360} B_{n p_{j}} \bar{F}_{n p_{j}}(C, G, M, Y)+\sum_{n=1}^{N} \frac{n-\zeta(n)}{360} B_{n} \pi_{n}(C, G, M, Y)}$.

Equation (45) provides us with a four parameter equity default swap pricing model and the parameters may be estimated from market prices of options on 
the underlying name. In this way we obtain option implied quotes for the equity default swap rates.

By way of a stylized example we set the interest rate yield curve at a flat $5 \%$ level and used the values $C=.5, G=2, M=10$, and $Y=.5$ with recovery at $50 \%$ and obtained the EDS prices for maturities of 1, 3, and 5 years at 161.97, 336.65 and 439.54 .

\section{CGMY Calibration to Option Data}

We obtained daily data on 5 year $C D S$ rates on Ford and GM over the period 25 February 2002 to 25 February 2005. There were 696 trading days in this time interval. The credit default swap rates on these names saw a sharp increase over this period before they finally came down again. The mean CDS rates over this period were 308 for Ford and 249 for GM. The standard deviations were 132 and 78 respectively while the minimum rates were 155 and 115 respectively. The corresponding maximal rates were 720 and 480 .

We employ the above algorithm to compute option implied equity default swap rates with barriers for the equity event set at the typical value of $30 \%$ of initiation price and a five year maturity. For this purpose we calibrate the $C G M Y$ model to European option prices for each day on each name. Equity option prices on Ford and GM traded on the New York Stock Exchange are typically available for American options. We mitigate the American feature by first employing out of the money options. Second we infer implied volatilities from American option prices using the Black Scholes model and then construct the corresponding European prices by the Black Scholes formula. The data is obtained from OptionMetrics and is available at WRDS the Wharton Research Data Service.

We note that the case $Y=0$ is the variance gamma model, that successfully calibrates any of the maturities. We are also aware that we may calibrate equally well with any specific value of the parameter $Y$. For our approximations to have a diffusion component we require $Y>0$ and the structure of the approximation is determined for any fixed value of $Y$. We therefore froze the value of the $Y$ parameter at $Y=.5$ and calibrated the parameters $C G M$ for this frozen value of $Y$. The calibrations were done using maturities between 1 and 2 years, by minimizing the root mean square error between market and model European option prices. We report in Table 1 summary statistics of the $C G M$ parameters for both the companies.

$\begin{array}{lllllll}\text { TABLE } & & & & & & \\ & \text { FORD } & & & \text { GM } & & \text { M } \\ & \text { C } & \text { G } & \text { M } & \text { C } & \text { G } & \text { Median } \\ .6506 & 1.9458 & 11.0187 & .2171 & 1.0084 & 5.8031 \\ 25 \% & .3661 & 1.3066 & 9.9309 & .1664 & .6690 & 4.7486 \\ 75 \% & 1.0895 & 4.0969 & 11.3522 & .5582 & 2.7802 & 11.5872\end{array}$


Figure 1: Ford CDS in Blue, EDS in Red

\section{$5 \quad$ Equity Default Swap Rates and the CDS Rates}

We employed the calibrated $C G M$ model with $Y=.5$ to determine the equity default swap rates for the typical barrier of $30 \%$ for the payout on the equity event. We present graphs of the implied EDS and CDS rates for both companies over the 2002-2005 period evaluated every five days. The data on $C D S$ rates for the five year maturity are readily available from Bloomberg.

The results of regressing the CDS rates on the EDS rates are as follows. We observe that there is a strong link between between the prices obtained from these separate markets.

$\begin{array}{lllllll} & \text { FORD } & & & \text { GM } & & \\ & \text { Const } & \text { Slope } & R^{2} & \text { Const } & \text { Slope } & R^{2} \\ \text { value } & 99.1820 & 0.2913 & 0.8950 & 146.2816 & 0.1442 & 0.6292 \\ t-\text { value } & 13.62 & 33.37 & & 18.10 & 14.89 & \end{array}$


Figure 2: GM CDS in Blue EDS in Red 


\section{Conclusion}

Approximation of the $C G M Y$ Lévy measure by hyperexponentials leads to an exact Wiener-Hopf factorization of the approximating process and hence to exact expressions for the first passage time of the approximating process to a level. We then employ these results to obtain closed form formulas for the prices of equity default swap contracts that payout on the equity event of a loss of $70 \%$ of the initial stock value with the receipts being a regular coupon paid till the equity event or maturity, whichever is less.

The methods are illustrated on $C G M Y$ processes calibrated to the vanilla options market for FORD and GM over the period 25 February 2002 to 25 February 2005. For the same period we also observe the daily values of the credit default swap contracts and these are compared favorably with the option imputed equity default swap prices computed by the $C G M Y$ approximation method proposed here. 


\section{References}

[1] Abate, J. and W. Whitt (1995), "Numerical Inversion of Laplace Transforms of Probability Distributions, ORSA, Journal of Computing, 7, 3643.

[2] Andersen, A. and B.F.Nielsen (1998), "A Markovian approach for modeling packet traffic with dependence," IEEE J. Sel. Areas Comm., 16, 719-732.

[3] Asmussen, S. (1992), "Phase-type representations in random walk and queueing problems," Annals of Probability, 20, 772-789.

[4] Asmussen, S., F. Avram and M. Pistorius (2004), "Russian and American put options under exponential phase-type Lévy models," Stochastic Processes and their Applications, 109, 79-111.

[5] Asmussen, S., M. Jobmann and H.P. Schwefel (2002), "Exact buffer overflow calculations for queues via martingales," Queueing Systems, 42, 63-90.

[6] Asmussen, S., O. Nerman and M. Olsson (1996), "Fitting phase-type distributions via the EM algorithm," Scandinavian Journal of Statistics, 23, 419-441.

[7] Asmussen, S. and J. Rosinski (2001), "Approximations of small jumps of Lévy prosesses with a view towards simulation," Journal of Applied Probability, 38, 2, 482-493.

[8] Boyarchenko, S.I. and Levendorskii (2000), "Option pricing for Truncated Lévy processes," International Journal for Theory and Applications in Finance, 3, 549-552.

[9] Campi, L. and A. Sbuelz (2005), "Closed-form pricing of Benchmark Equity Default Swaps under the CEV assumption," Working Paper, Finance Department, Tilburg University.

[10] Carr, P., H. Geman, D. Madan, and M. Yor (2002), "The Fine Structure of Asset Returns: An Empirical Investigation," Journal of Business, 75, 2, 305-332.

[11] Chiu, S.N. and C. Yin (2005), "Passage times for a spectrally negative Levy process with applications to risk theory," Bernoulli, 11, 511-522.

[12] Davydov, D. and V. Linetsky (2001), "Pricing and hedging path dependent options under the CEV process," Management Science, 47, 949-965.

[13] Feldman, A. and W. Whitt (1998), "Fitting Mixtures of Exponentials to long-tailed distributions to analyze performance models," Performance Evaluation, 31, 245-279.

[14] Khanna, A. and D. Madan (2004), "Understanding Option Prices," Quantitative Finance, 4, 55-63. 
[15] Koponen, I. (1995), "Analytic Approach to the problem of convergence of truncated Lévy flights towards the Gaussian stochastic process," Physical Review E52, 1197-1199.

[16] Nguyen-Ngoc, L. and M. Yor (2002), "Exotic Options and Lévy Processes," to appear in Eds. Yacine Aït Sahalia and Lars Peter Hansen, Handbook of Financial Econometrics, North Holland.

[17] Novikov, A., R.E. Melchers, E. Shinjikashvili and N. Kordzakhia (2003), "First Passage Times of Filtered Poisson Process with Exponential Shape Function," Working Paper, University of Technology, Sydney.

[18] Rogers, L.C.G. (2000), "Evaluating first-passage probabilities for spectrally one-sided Lévy processes," Journal of Applied Probability, 37, 1173-1180. 


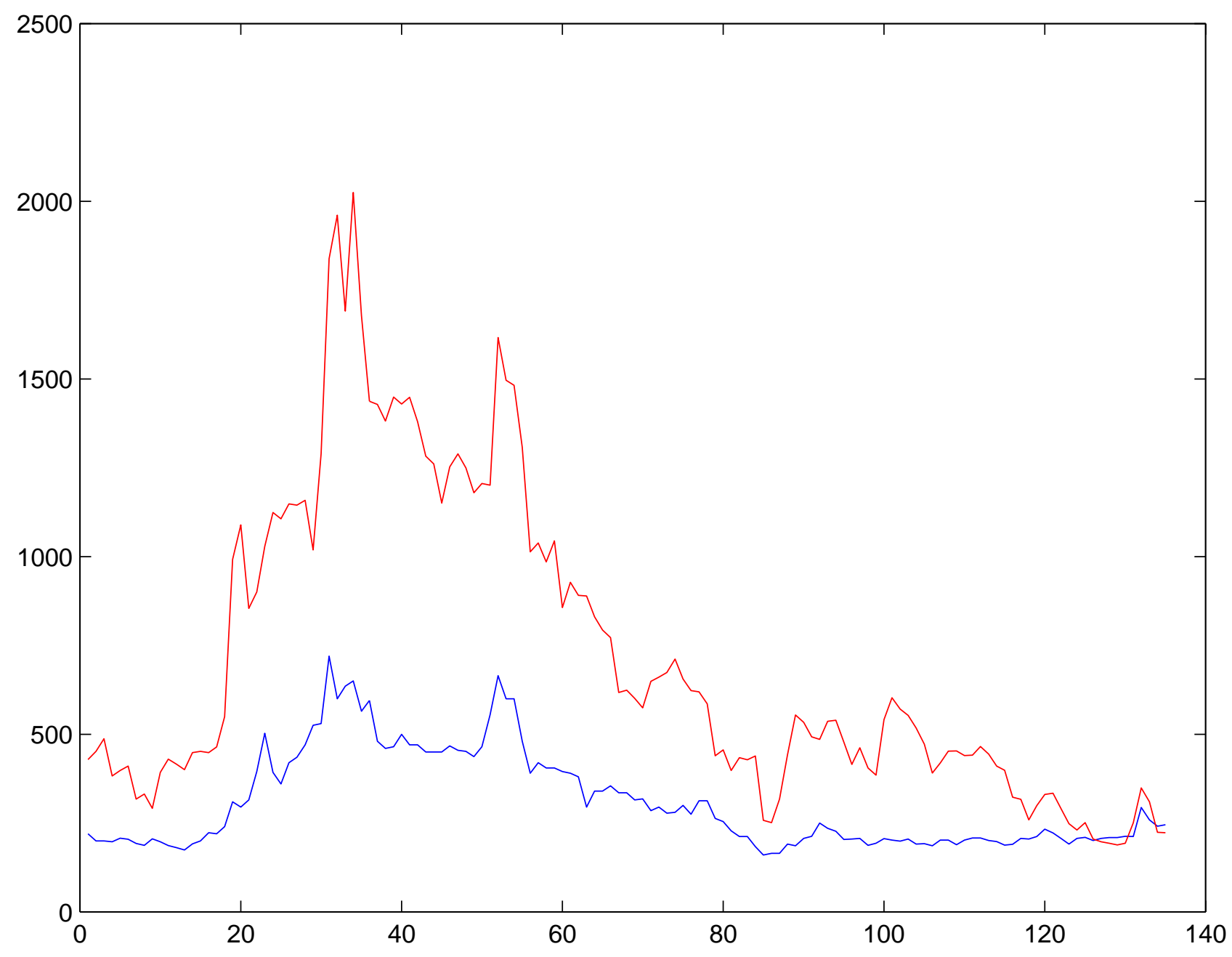




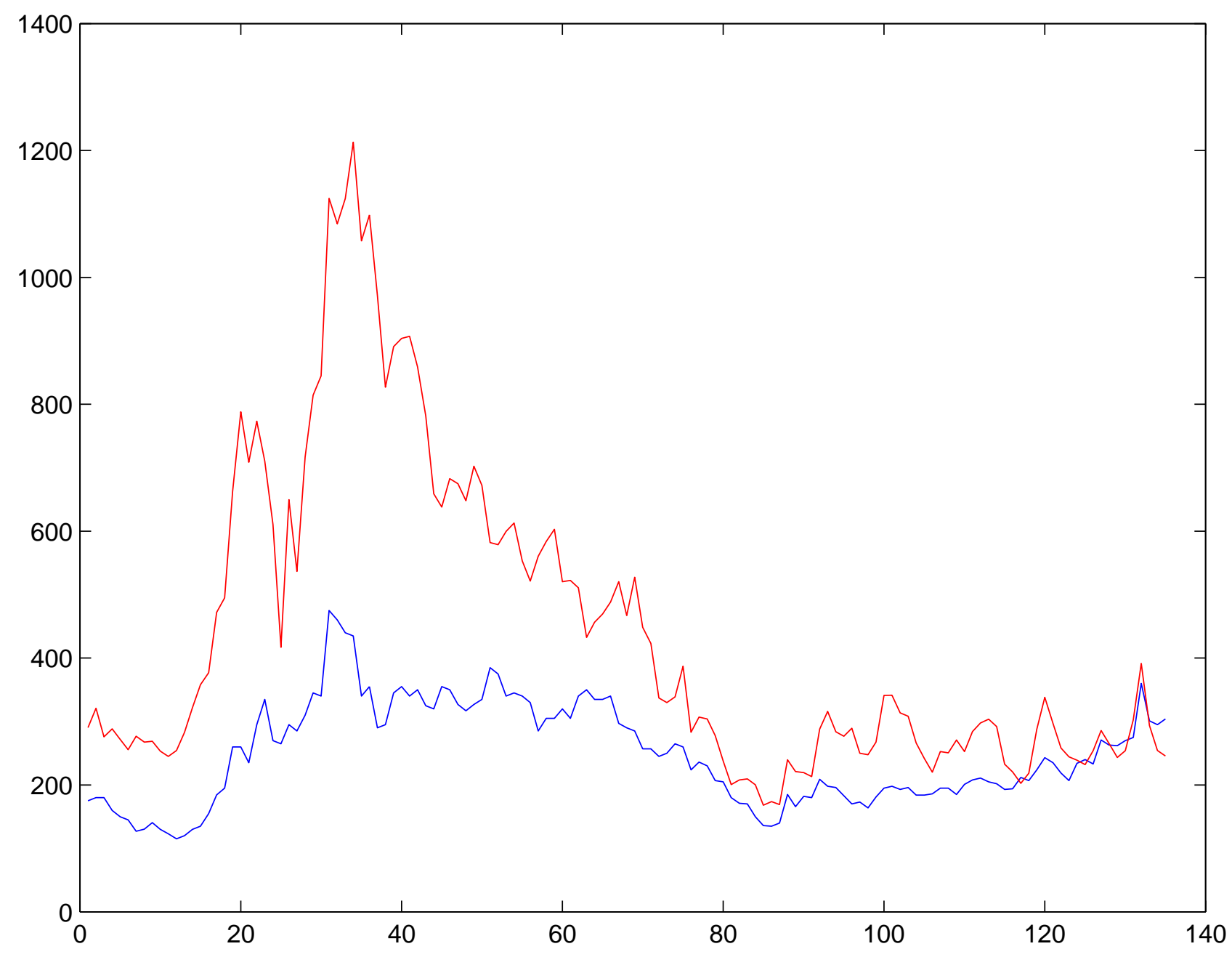

